\title{
Looking for Pets: um jogo de estratégia para o desenvolvimento do Pensamento Computacional
}

\author{
Luiz Alencar ${ }^{1}$, Marcela Pessoa ${ }^{1}$, Fernanda Pires $^{1}$ \\ ${ }^{1}$ Escola Superior de Tecnologia - Universidade do Estado do Amazonas (UEA) \\ 69050-020 - Manaus - AM - Brazil \\ \{lfba.lic17, msppessoa, fpires\}@uea.edu.br
}

\begin{abstract}
This article introduces "Looking for Pets," a strategy game that exercises decision making and solving algorithms. The game was developed for mobile platforms and aims to assist in the development of Computational Thinking through a playful process for the creation of algorithms. The game uses with theoretical support of the Constructionist Theory. Preliminary tests point out that it can act in the pedagogical field generating positive impacts for learning.

Resumo. Este artigo apresenta o "Looking for Pets", um jogo de estratégia que exercita tomadas de decisão e através da resolução de algoritmos. O jogo foi desenvolvido para plataformas móveis e tem como objetivo auxiliar no desenvolvimento do Pensamento Computacional através de um processo lúdico para a criação de algoritmos. O jogo usa com suporte téorico da aprendizagem a Teoria Construcionista. Testes preliminares apontam que o mesmo pode agir no campo pedagógico gerando impactos positivos para aprendizagem.
\end{abstract}

\section{Cenário de Uso}

Pensamento Computacional (PC) diz respeito a um conjunto de habilidades que permitem resolver problemas da melhor forma possível, sendo estas necessárias em todas as áreas do conhecimento e não somente nas áreas relacionadas à Computação. Por esse motivo, acredita-se que o Pensamento Computacional deveria ter um espaço no currículo escolar tal qual Linguística e Matemática [Wing 2006].

A Prova Brasil é um dos principais instrumentos para avaliar a proficiência de estudantes da educação infantil no território nacional. Pesquisas revelam que apenas $11 \%$ dos estudantes do $9^{\circ}$ ano apresentam a proficiência esperada em Matemática [INEP/MEC 2017]. Considerando que o Pensamento Computacional é fundamental para o desenvolvimento das habilidades e competências, através do pensamento crítico aplicado a soluções criativas, relações lógicas e matemáticas aplicadas, entrelaçamento de saberes, entre outros [Brackmann et al. 2017], investir em iniciativas que ajudem a desenvolver as habilidades do Pensamento Computacional podem ajudar a melhorar o desempenho dos estudantes.

Neste contexto, este artigo apresenta Looking for Pets, um jogo de estratégia com mecânicas de puzzles, gráfico em 3D, para plataformas móveis.O jogo usa realidade aumentada para estimular as habilidades do Pensamento Computacional, em que os jogadores devem elaborar diferentes tipos de algoritmos para conseguir avançar nas etapas. $\mathrm{O}$ jogo é uma ventura lógica e seu público-alvo são crianças com faixa etária entre 10 e 13 anos. 
VIII Congresso Brasileiro de Informática na Educação (CBIE 2019)

Anais dos Workshops do VIII Congresso Brasileiro de Informática na Educação (WCBIE 2019)

\section{Desenvolvimento}

O jogo Looking for Pets foi construído na engine de desenvolvimento Unity. O Unity é uma ferramenta utilizada para produção de jogos, destinada a aplicações $2 \mathrm{D}$ e 3D, com códigos fontes na linguagem de programação C\#, voltada para projetos de jogos e aplicativos destinados a dispositivos móveis, desktops, consoles e outras plataformas. O Looking for Pets foi projetado para ser utilizado em smartphones com Sistema Operacional Android com recomendação nas versões Android 6.0 ou superior.

Para o desenvolvimento do jogo foram seguidas as etapas apresentadas no diagrama da Figura 1, conforme descrito a seguir:

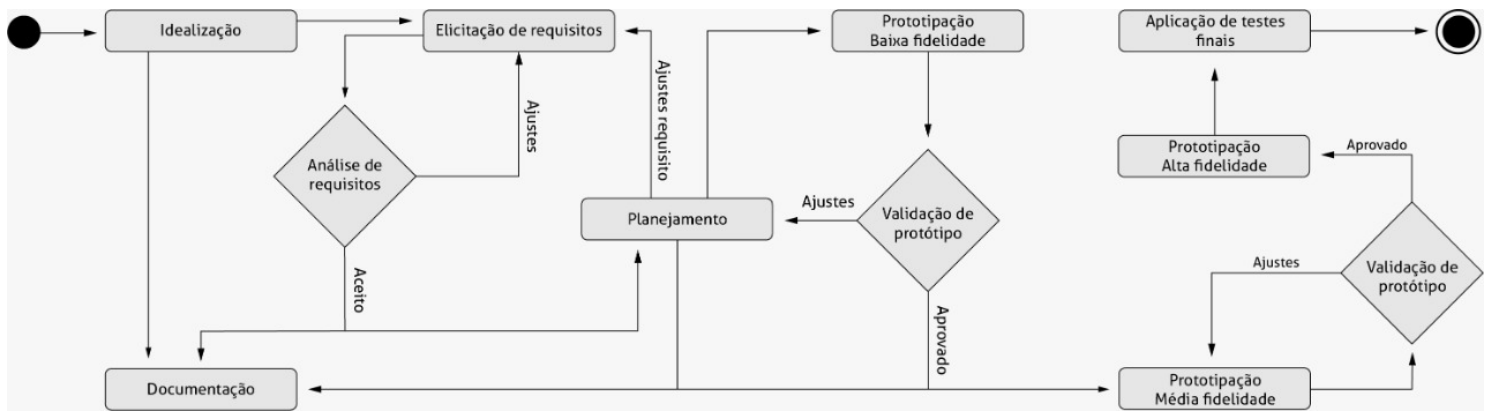

Figura 1. Diagrama das etapas de desenvolvimento do jogo

- Idealização: nesta etapa foram concebidas as primeiras ideias do jogo, incluindo a identificação dos principais elementos e características, tal como o formato de gameplay e mecânicas, além de fazer um rascunho inicial da proposta visual;

- Elicitação de requisitos: foram levantados os requisitos para o desenvolvimento do jogos, entre eles os requisitos funcionais e não funcionais, além dos aspectos pedagógicos e teoria cognitiva da aprendizagem;

- Análise de requisitos: nesta etapa os requisitos foram analisados gerando as funcionalidade e restrições. Foram necessárias novas discussões que resultaram em novos requisitos e adaptações dos requisitos anteriores;

- Planejamento: nesta fase foi realizada uma adaptação do Game Design Document (GDD). Nele são apresentadas informações detalhadas do jogo, tais como: descrição de elementos da gameplay e mecânicas, características das fases, controles do jogador, sons e efeitos sonoros no ambiente, detalhes do heads-up display (HUD), entre outras;

- Protótipo de Baixa Fidelidade: nesta etapa foi desenvolvido um modelo prévio de prototipagem visual do jogo, evidenciando a organização dos elementos das telas e funcionalidades do jogo. Após cada etapa de prototipação é feita a validação do protótipo, etapa descrita posteriormente;

- Protótipo de Média Fidelidade: o protótipo de média fidelidade seguiu as diretrizes presentes no GDD durante a implementação do jogo, seguindo as informações descritas na documentação. Nessa etapa foi utilizada a engine Unity para a implementação.

- Protótipo de Alta Fidelidade: o protótipo de alta fidelidade consiste na implementação de melhorias no jogo, levando em consideração os resultados dos testes desenvolvidos na etapa de Validação, em que apresenta novas funcionalidades e um aumento no número de fases. 
VIII Congresso Brasileiro de Informática na Educação (CBIE 2019)

Anais dos Workshops do VIII Congresso Brasileiro de Informática na Educação (WCBIE 2019)

- Validação de protótipo: a cada fim das etapas de protótipo baixa, média e alta fidelidade o aplicativo é avaliado para verificar se as funcionalidades implementadas estão de acordo com os requisitos definidos. A validação após protótipo de baixa e média fidelidade é realizada pelos desenvolvedores. Após o protótipo de alta fidelidade é realizada a avaliação das interfaces utilizando o Teste de Usabilidade de Nielsen [Nielsen 1994] que também foi executado pelos desenvolvedores.

- Aplicação de testes finais: nesta etapa são realizados testes com o usuário. O aplicativo proposto é validado com os usuários finais utilizando o teste MEGA+KIDS [von Wangenheim et al. 2018], cujo objetivo é avaliar jogos com propósito educacional para aprendizagem de conceitos relacionados à computação. No caso do jogo proposto, participaram do teste 20 crianças de uma escola pública de [oculto para revisão], com faixa etária entre dez e treze anos.

- Documentação: durante todas as etapas do processo de desenvolvimento são gerados artefatos de documentação, entre eles o GDD.

\section{Apresentação do software}

Looking For Pets é um jogo de estratégia com personagens de traços geométricos. O cenário do jogo é uma cidade pequena que sofre uma erupção vulcânica. Lara, personagem principal, é estudante de Medicina Veterinária e está responsável por cuidar dos animais que vivem no centro comunitário. Com o abalo, os animais fugiram assustados. A missão de Lara é resgatar os animais até que todos estejam protegidos. Com vários obstáculos dificultando o percurso até cada animal, o jogador deve construir a sequência de passos necessários para chegar até cada animal.

O jogo foi projetado para ter grau de dificuldade crescente. A primeira fase tem como objetivo apresentar o cenário e as possíveis ações do jogador e, de acordo com o avanço das fases, aumentam as dificuldades do jogador para alcançar os animais. $\mathrm{O}$ desempenho do jogador é medido através da quantidade de pontos que Lara alcança nas fases. A pontuação é representada por estrelas e cada fase do jogo possui no máximo três estrelas.

\subsection{Mecânica do Jogo}

O jogador deve montar uma sequência de ações (algoritmo) que Lara deve realizar para chegar ao animal. Entre as ações possíveis estão ir para frente, virar para a direita, virar para a esquerda e pular. Após definidos os passos, o jogador seleciona a opção para executar o algoritmo e todos os passos são executados em uma única vez, seguindo a sequência definida pelo jogador.

Para facilitar a visão do jogador é possível rotacionar a tela em um ângulo de 360 graus utilizando o touchscreen do dispositivo. A cada fase o jogador recebe estrelas que permitem desbloquear novos estilos visuais para Lara.

\subsection{Descrição das Telas}

Esta seção apresenta as telas e uma breve descrição destas no jogo. A primeira tela, Figura 2, é a apresentação do jogo e contêm os botões de "iniciar"e "configurações". Na tela de configurações, Figura 3, os jogadores podem desativar e ativar os sons do jogo além de apresentar o botão de "créditos", Figura 4, e de "informações", Figura 5, que apresenta o propósito do jogo. 
VIII Congresso Brasileiro de Informática na Educação (CBIE 2019)

Anais dos Workshops do VIII Congresso Brasileiro de Informática na Educação (WCBIE 2019)

Ao clicar em "iniciar"o jogador é direcionado para a tela de seleção de fases (Figura 6), que permite optar por iniciar uma partida ou selecionar um estilo visual para Lara, se disponível. Após a escolha da fase é apresentada a tela de gameplay (Figura 7).

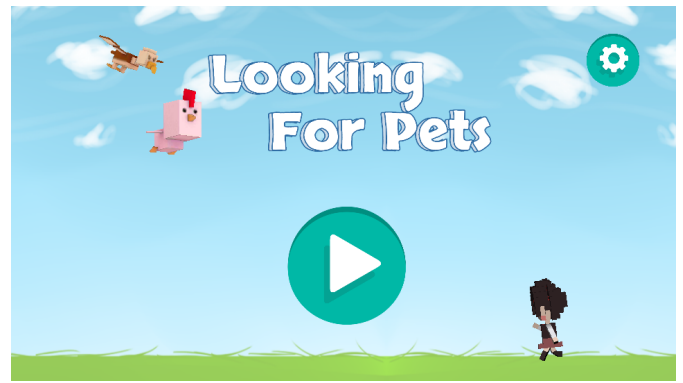

Figura 2. Tela de menu principal

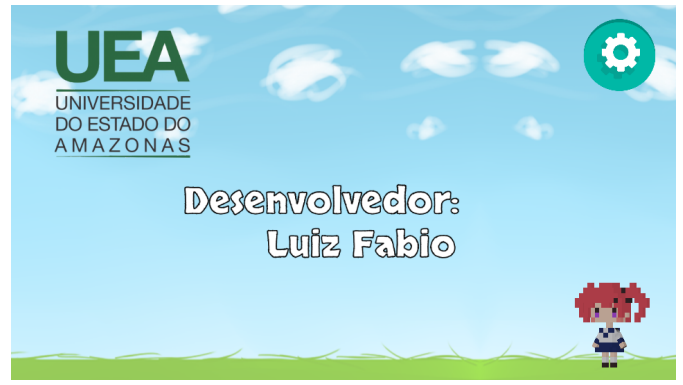

Figura 4. Tela de créditos

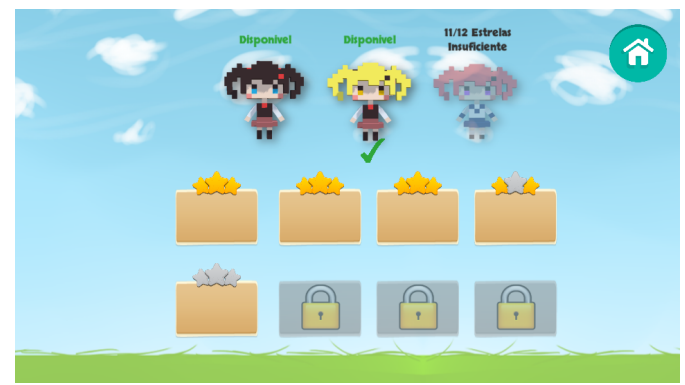

Figura 6. Tela de seleção de fases

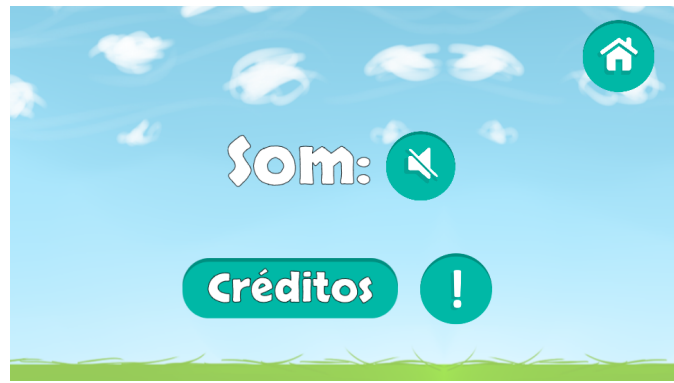

Figura 3. Tela de configurações

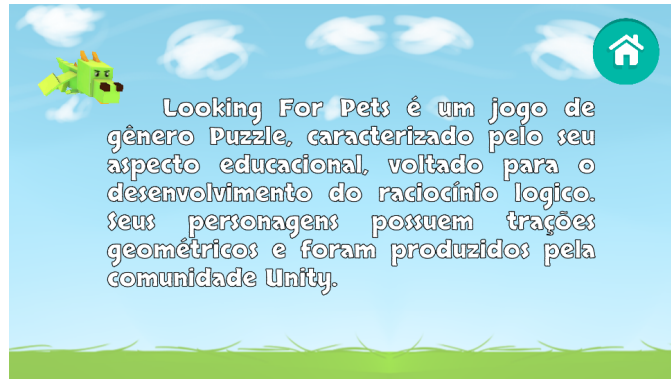

Figura 5. Tela de informações

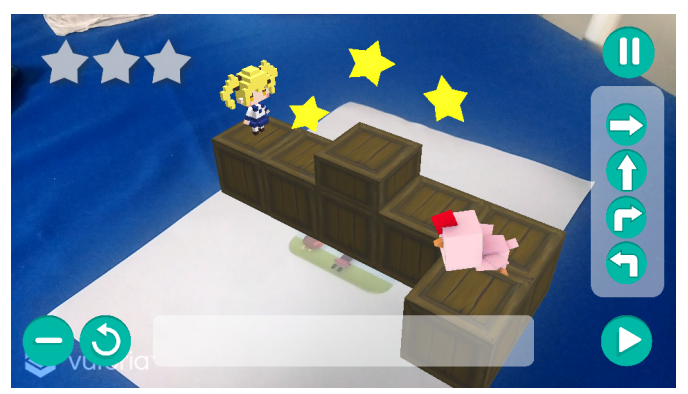

Figura 7. Tela de gameplay

Para jogar, a câmera do dispositivo deve identificar a imagem de marcação, representada pela Figura 8, para então ser reproduzida a fase. Caso a câmera do dispositivo não identifique a imagem, o jogo não reproduz o ambiente da fase selecionada e é levado a uma tela de feedback solicitando que o usuário foque a câmera na imagem, conforme ilistra a Figura 9.

Para uma melhor visualização da estrutura e conexão das telas, a Figura 10 apresenta os possíveis estados do aplicativo. Nela é possível observar o fluxo do jogo, bem como a transição geral das principais telas. A partir do Menu Principal, o usuário pode ir para Configurações, Informações ou Selecionar uma das fases. Na opção Seleção de Fases, caso o usuário não tenha desbloqueado nenhuma fase, ele é direcionada para a primeira e as demais se mantém bloqueadas. À medida que o jogador vai desbloqueando fases vai podendo selecionar entre as fases já desbloqueadas. 
VIII Congresso Brasileiro de Informática na Educação (CBIE 2019)

Anais dos Workshops do VIII Congresso Brasileiro de Informática na Educação (WCBIE 2019)
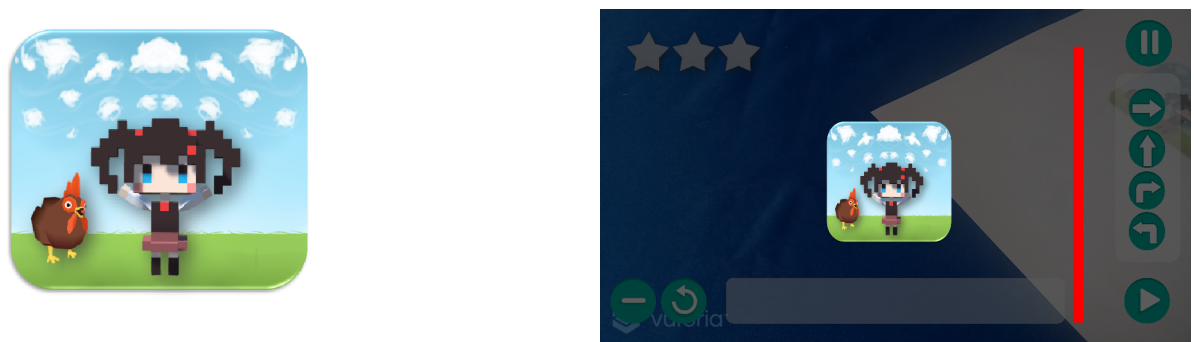

Figura 8. Imagem de marcação

Figura 9. Imagem não identificada

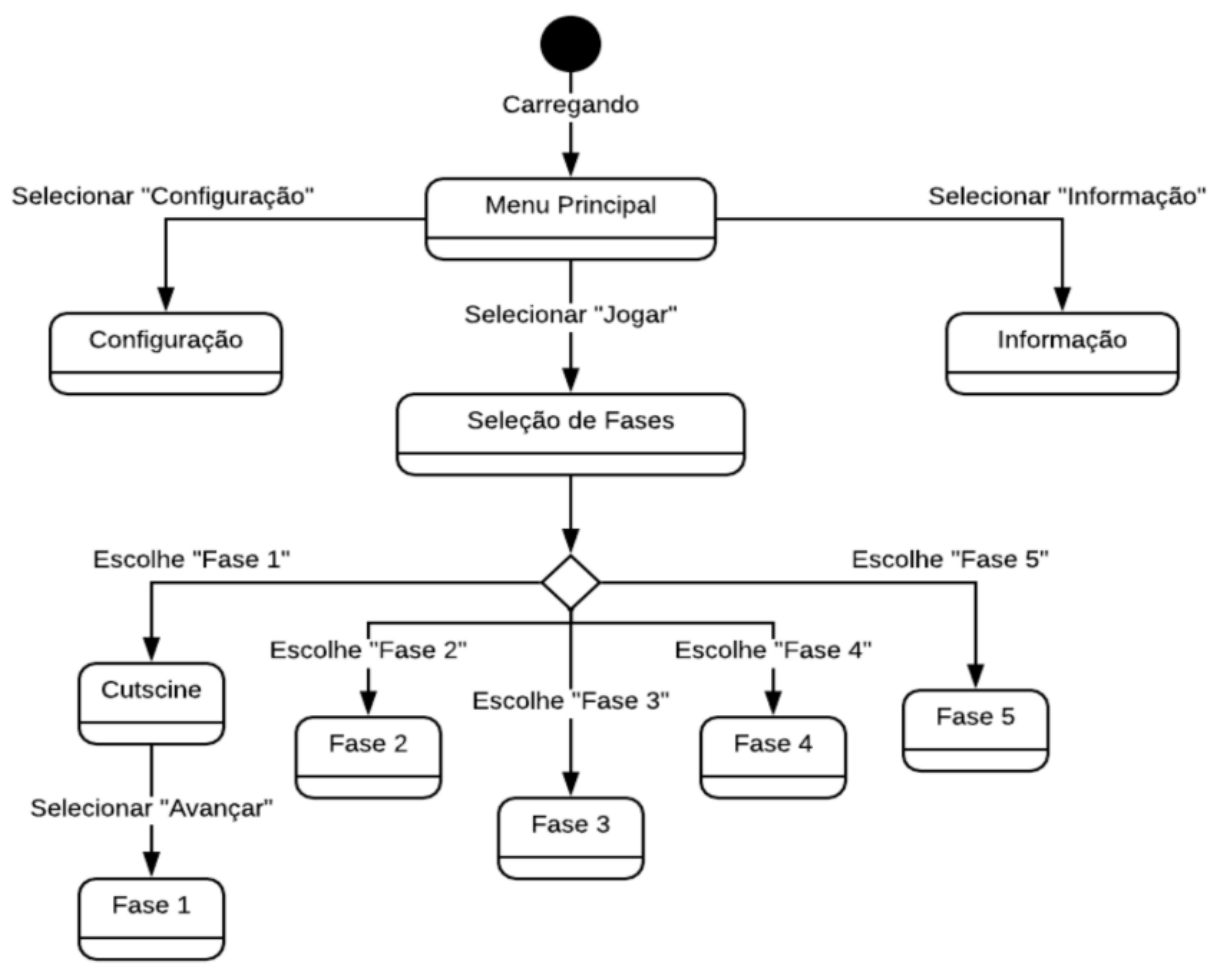

Figura 10. Diagrama de telas 
VIII Congresso Brasileiro de Informática na Educação (CBIE 2019)

Anais dos Workshops do VIII Congresso Brasileiro de Informática na Educação (WCBIE 2019)

\section{Considerações finais}

Este artigo apresenta a proposta de um jogo denominado Looking for Pets. É um jogo no estilo estratégia com mecânicas de puzzle, com personagens de traços geométricos. $\mathrm{O}$ jogo tem como objetivo auxiliar no desenvolvimento do Pensamento Computacional peo exercício do raciocínio lógico através da definição de um conjunto de passos que levam a personagem ao objetivo. Entre as ações possíveis está: ir para a frente, virar à direita, virar à esquerda e pular.

Como forma de validação, o jogo foi testado com crianças entre dez e treze anos, através do teste MEGA+KIDS, excluindo os critérios Acessibilidade e Interação. Neste jogo o MEGA+KIDS mediu critérios como Estética, Aprendizagem, Controle, Desafio, Satisfação, Atenção e Relevância seguindo uma escala de notas de 0 a 5. No caso do jogo proposto, as notas com predominância foram de 5 e 4 .

Um outro teste que foi aplicado foi o teste de Usabilidade de Nielsen. Ele apresenta dez heurísticas e os resultados são que o jogo atende oito delas. Entre as heurísticas mais bem avaliadas está "Controle do usuário e liberdade"e a pior avaliada é "Prevenção de erros". Em trabalhos futuros serão melhorados os pontos relacionados às heurísticas e desenvolvidas novas fases.

\section{Referências}

Brackmann, C., Boucinha, R. M., Roman-Gonzalez, M., Barone, D. A. C., and Casali, A. (2017). Pensamento computacional desplugado: Ensino e avaliacao na educacao primaria espanhola. In Anais dos Workshops do Congresso Brasileiro de Informática na Educação, volume 6, page 982.

INEP/MEC (2017). Distribui $\tilde{A} \S \tilde{A} £ o$ dos alunos por $n \tilde{A} v e l$ de profici $\tilde{A}^{a}$ ncia.

Nielsen, J. (1994). Usability inspection methods. In Conference companion on Human factors in computing systems, pages 413-414. ACM.

von Wangenheim, C. G., Petri, G., and Borgatto, A. F. (2018). Meega+ kids: A model for the evalua-tion of educational games for compu-ting education in secondary school.

Wing, J. M. (2006). Computational thinking. Communications of the ACM, 49(3):33-35. 